\title{
Criteria used in the specifications for geographical indications of fine wines in Brazil / Critères utilisés dans les cahiers des charges des indications géographiques des vins fins au Brésil
}

\author{
Jorge Tonietto $^{1, \text { a }}$, Rafaela Vieira Pereira ${ }^{2}$, Mauro Celso Zanus ${ }^{1}$, Celito Crivellaro Guerra ${ }^{1}$, et Ivanira Falcade ${ }^{3}$ \\ ${ }^{1}$ Embrapa Grape and Wine, Rua Livramento, 515, 95701-008 Bento Gonçalves, RS, Brazil \\ ${ }^{2}$ Universidade Federal Fluminense, 27255-250 Volta Redonda, RJ, Brazil \\ ${ }^{3}$ Universidade de Caxias do Sul, Alameda João Dal Sasso, 800, 95705-266 Bento Gonçalves, RS, Brazil
}

\begin{abstract}
The Brazilian legislation on geographical indications (GI) are the Law 9.279 and INPI Normative Instruction 25/2013, establishing the conditions for the registration of GI, whether in modality of "Indicação de Procedência" (IP) or "Denominação de Origem" (DO). Brazil has recognized some GI of fine wines - IP Vale dos Vinhedos, Pinto Bandeira, Altos Montes, Monte Belo and Farroupilha, and DO Vale dos Vinhedos. Others are in structuring process - Campanha Gaúcha, Vale do São Francisco and Altos de Pinto Bandeira. Even without detailing, by INPI, the elements that must be in the specification document, the GI already recognized include, among others: delimited geographical area; varieties of Vitis vinifera authorized; grape production in the delimited area; training systems; maximum productivity; quality of grapes for wine making; authorized wines; oenological practices; physicochemical characteristics for each type of wine and mandatory sensory analysis; elaboration, aging and bottling in the delimited area; labeling standards. In addition, for each GI there is a specific plan of control. It is possible to observe that the specifications for Brazilian GI of wines have some similarity to those adopted in the European Community, and are more specific and demanding than most of the existing GI in the New World.
\end{abstract}

\section{Introduction}

La législation brésilienne sur les indications géographiques (IG) a été créée par la Loi de la Propriété Industrielle n. 9.279, de 1996 [1]. L'Instruction Normative n. 25/2013 de l'Institut National de la Propriété Industrielle - INPI [2], établie les conditions pour le registre des indications géographiques, que ce soit pour la «Indicação de Procedência 》 (IP) ou pour la « Denominação de Origem » (DO). Depuis 2002, ont été reconnues quelques indications géographiques des vins fins brésiliens, les IP Vale dos Vinhedos, Pinto Bandeira, Montes Altos, Monte Belo et Farroupilha, et la DO Vale dos Vinhedos. Autres IG sont en processus d'structuration - IP Campanha Gaúcha, IP Vale do São Francisco et DO Altos de Pinto Bandeira.

L'objectif de l'article est d'analyser les spécifications de contenu technique des cahiers des charges des IG de vins fins brésiliens reconnues, avec les normatives légales exigés pour le registre d'une IG au Brésil.

\section{Exigences pour le registre d'une IG au Brésil}

Tout d'abord, la loi brésilienne établie que constitue indication géographique la «Indicação de Procedência» et la «Denominação de Origem » [1], avec les définitions suivantes :

On considère «Indicação de Procedência» le nom géographique de pays, ville, région ou localité de

a e-mail: jorge.tonietto@embrapa.br son territoire, qui est devenue connu comme centre d'extraction, production ou fabrication d'un produit déterminé ou de prestation d'un service déterminé;

On considère « Denominação de Origem 》 le nom géographique de pays, ville, région ou localité de son territoire, qui désigne produit ou service dont les qualités ou caractéristiques sont dues exclusivement ou essentiellement au milieu géographique, inclus les facteurs naturels et humains.

Pour la demande de registre d'une IG, l'Instruction Normative n. 25/2013 de l'Institut National de la Propriété Industrielle - INPI [2], exige la présentation du cahier des charges de l'IG, appelé « regulamento de uso», sans spécification de son contenu.

D'autres éléments doivent intégrer le dossier de demande de registre comme, entre autres : le nom géographique de l'IG, la description du produit ou service, l'instrument officiel de délimitation de l'aire géographique, document de preuve de l'existence d'une structure de contrôle sur les producteurs ou prestataires de services que auront le droit d'usage exclusif de l'IG, bien que sur le produit ou la prestation du service distingué avec l'IG ; documents de preuve que les producteurs ou prestataires de services soient bien établies dans l'aire géographique démarqué et en exercent, effectivement, les activités de production ou prestation du service.

En plus, pour une IP est exigé : des documents de preuve que le nom géographique est devenu connue (renommé) comme centre d'extraction, production ou fabrication du produit ou de la prestation de service. 
En plus, pour une DO est exigé : les éléments qui identifient l'influence du milieu géographique sur la qualité ou caractéristiques du produit ou service, qui soient dues exclusivement ou essentiellement au milieu géographique, inclus les facteurs naturels et humains; la description du processus ou méthode d'obtention du produit ou service, lesquels doivent être locales, loyales et constants.

\section{Les spécifications des cahiers des charges des IG des vins fins brésiliennes}

Une analyse a été réalisée sur les cahiers des charges des IG de vins fins brésiliens reconnues : IP Pinto Bandeira, Montes Altos, Monte Belo et Farroupilha, et DO Vale dos Vinhedos [3-7].

Tous les cahiers des charges spécifient les éléments suivants :

- le nom géographique de l'IG et la description précise de l'aire géographique délimitée ;

- les cépages de Vitis vinifera autorisés - dans certaines IG sont spécifies les cépages par type de produit et composition, en pourcentage, maximale ou minimale de chaque cépage par type de produit ;

- la production du raisin dans la zone délimitée entre 85 à $100 \%$ pour les IP et $100 \%$ pour la DO ;

- le (s) système (s) de conduite autorisés - pour les IP, parfois ce sont l'ensemble des systèmes de la zone, parfois est autorisé un seul système (espalier, par exemple, dans le cas de la DO) ;

- la productivité maximale/hectare - toujours présente, la limite maximale de productivité par hectare est établie parfois avec des particularités liées au cépage autorisée et/ou type de produit ; pour la DO, en plus de la limite par hectare, il y a également une limite de production par plante et par type de produit ;

- la qualité du raisin pour la vinification - spécification liée surtout à la teneur en sucres minimale ou au potentiel alcoolique naturel du vin ;

- les produits vinicoles autorisés - relation du ou des produits autorisés (ex. : vin fin blanc sec, vin fin rouge sec);

- les pratiques œnologiques - qui spécifient quelques pratiques autorisées ou interdites pour les vins IG, dans ce dernier cas, interdites même si elles sont autorisées par la loi nationale du vin ;

- les caractéristiques physico-chimiques des vins - en plus d'obéir aux caractéristiques physico-chimiques définies de la loi nationale du vin, il existe quelques caractéristiques physico-chimiques plus restreintes établies par type de vin dans chaque IG, pour assurer une qualité différentielle au vin ;

- les caractéristiques sensorielles du vin - en plus d'obéir les caractéristiques sensorielles de la loi nationale du vin, il y a des analyses sensorielle obligatoires sur $100 \%$ des vins IG, pour vérifier si les vins n'ont pas des défauts visuels, olfactifs ou gustatifs, si les caractéristiques sont présents pour les vins de cépage et si les caractéristiques sensoriels de typicité sont présentes dans le cas des vins avec DO ;
- l'élaboration, la maturation et l'embouteillage de vins dans l'aire géographique délimitée - établie comme obligatoire pour les IP reconnues, avec éventuels exceptions pour quelques types de vins, en ce qui concerne une ou autre pratique dans la zone de proximité immédiate pour les IP ; obligatoire en cas de DO ;

- les normes d'étiquetage - présentes pour toutes les IG, avec l'indentification, dans l'étiquette principale du vin, plus avec un signe de chaque IG, avec une numérotation unique pour chaque bouteille envisageant d'assurer la traçabilité du produit IG ;

- les contrôles - chaque cahier des charges possède des éléments du système de contrôle et de la normative de contrôle pour assurer le respect du cahier des charge, l'attestation de la conformité du produit ou les sanctions.

Chaque IG possède également un plan de contrôle spécifique [8]. Dans les IG reconnues actuellement, le système comprenne l'autocontrôle et le contrôle interne qui est géré par de «Conselho Regulador» de chaque association qui est chargé de la gestion et défense de l'IG.

Les IG de vins fins brésiliennes reconnues ont y les projets d'structuration coordonnés par l'Embrapa Uva $e$ Vinho - institution neutre de recherche technologique et développement, qui adopte, par une manque de spécifications plus précises pour les IG de vins et, même sans valeur normatif obligatoire, une charte institutionnelle d'orientation, qui comprenne également des recommandations pour les cahiers des charge [9]. Cela explique en partie pourquoi les IG ont une certaine uniformité de contenu dans les cahiers des charges, tout en valorisant les originalités de chaque IG. Les cahiers des charges sont disponibles sur le site d'Embrapa Uva e Vinho [10].

\section{Des points communs entre les cahiers des charges des IG de vins du Brésil et de la CE}

Une analyse comparative des éléments de contenu des cahiers des charges des IG de vins fins du Brésil et les éléments définies comme minimales au niveau de la Communauté Européenne [11,12], permet de conclure qu'il existe une certaine ressemblance de contenu. Une partie des exigences des cahiers des charges de la CE (éléments du milieu géographique, qualité et caractéristiques des produits, lient de causalité entre les deux, renommé), au Brésil font partie de la documentation de demande de reconnaissance, pas forcément inclus dans les cahiers des charges. L'inclusion en 2007, de la part de la CE de l'IG Vale dos Vinhedos dans la relation des pays tiers avec IG de vin, déjà, un niveau d'équivalence.

De cette analyse, on conclue également que les exigeantes et spécifiques des cahiers des charges des IG au Brésil sont plus strictes que celles adoptées dans la plupart des IG des pays du Nouveau Monde.

\section{Références}

[1] Brasil, Lei n 9.279, de 14 de maio de 1996, (Diário Oficial [da] República Federativa do Brasil, Brasília, DF, 15 maio 1996. (Seção 1, p. 8353-8366, 1996) 
[2] INPI, Instituto Nacional da Propriedade Industrial. Instrução Normativa $n .^{\circ}$ 25/2013 de 21 de agosto de 2013. (INPI, Rio de Janeiro, 2013)

[3] Embrapa, Regulamento de uso da Indicação de Procedência região de Monte Belo, (Embrapa Uva e Vinho, Bento Gonçalves, 2012). (Nota técnica. Parte do projeto SEG 02.05.0.15.00.00, Convênio Finep Ref. 0452/2008 com a sigla APL Vinhos)

[4] Embrapa, Regulamento de uso da Indicação de Procedência Farroupilha, (Afavin, Farroupilha, 2014). (Parte do projeto Embrapa SEG 04.08.08.001.00.00, Edital 08/2008, Macroprograma 4, Sigla IG Vinhos)

[5] J. Tonietto, M. C. Zanus, I. Falcade, C. C. Guerra, $O$ regulamento de uso da indicação geográfica Pinto Bandeira: vinhos finos tranquilos e espumantes (Embrapa Uva e Vinho, Bento Gonçalves, 2013). (Embrapa Uva e Vinho. Documentos, 83)

[6] J. Tonietto, M. C. Zanus, I. Falcade, C. C. Guerra, $O$ regulamento de uso da denominação de origem Vale dos Vinhedos: vinhos finos tranquilos e espumantes (Embrapa Uva e Vinho, Bento Gonçalves, 2013). (Embrapa Uva e Vinho. Documentos, 84)

[7] J. Tonietto, M. C. Zanus, I. Falcade, C. C. Guerra, $O$ regulamento de uso da indicação geográfica
Altos Montes: vinhos finos tranquilos e espumantes (Embrapa Uva e Vinho, Bento Gonçalves, 2013). (Embrapa Uva e Vinho. Documentos, 85)

[8] Embrapa, Normas, de controle da Denominação de Origem Vale dos Vinhedos (Embrapa Uva e Vinho, Bento Gonçalves, 2010). (Resultados do projeto código SEG 02.05.0.15.00.00, Convênio Finep 01.09.0494.00, Sigla: APL Vinhos)

[9] Embrapa, Projetos de indicações geográficas de vinhos: referenciais do "IG padrão Embrapa Uva e Vinho" (Embrapa Uva e Vinho, Bento Gonçalves, 2014)

[10] Embrapa, Indicações geográficas de vinhos finos do Brasil (Embrapa uva e Vinho, Bento Gonçalves, 2013) (Disponível em: http://www. cnpuv.embrapa.br/tecnologias/ig/. Acesso em: 17 ago. 2016)

[11] União Europeia, Regulamento (CE) $n^{\circ} 479 / 2008$ de 29 de abril de 2008, Jornal Oficial da União Europeia, n. L 148, p.1-61, 06 jun. (2008)

[12] União Europeia, Regulamento (CE) $N^{\circ}$ 607/2009 Da Comissão de 14 de Julho de 2009, Jornal Oficial da União Europeia, n.L 193 p. 60-139, 24 jul. (2009) 